\title{
Gradhiva
}

GRADHIV

Revue d'anthropologie et d'histoire des arts

9 | 2009

Arts de l'enfance, enfances de l'art

\section{Des enfants dessinateurs au Moyen Âge}

\section{René Baldy et Daniel Fabre}

\section{OpenEdition}

\section{Journals}

Édition électronique

URL : http://journals.openedition.org/gradhiva/1440

DOI : $10.4000 /$ gradhiva. 1440

ISSN : 1760-849X

\section{Éditeur}

Musée du quai Branly Jacques Chirac

\section{Édition imprimée}

Date de publication : 2 septembre 2009

Pagination : 152-163

ISBN : 978-2-35744-009-8

ISSN : 0764-8928

Référence électronique

René Baldy et Daniel Fabre, "Des enfants dessinateurs au Moyen Âge », Gradhiva [En ligne], 9 | 2009, mis en ligne le 02 septembre 2012, consulté le 01 mai 2019. URL : http://journals.openedition.org/ gradhiva/1440; DOl : 10.4000/gradhiva.1440 


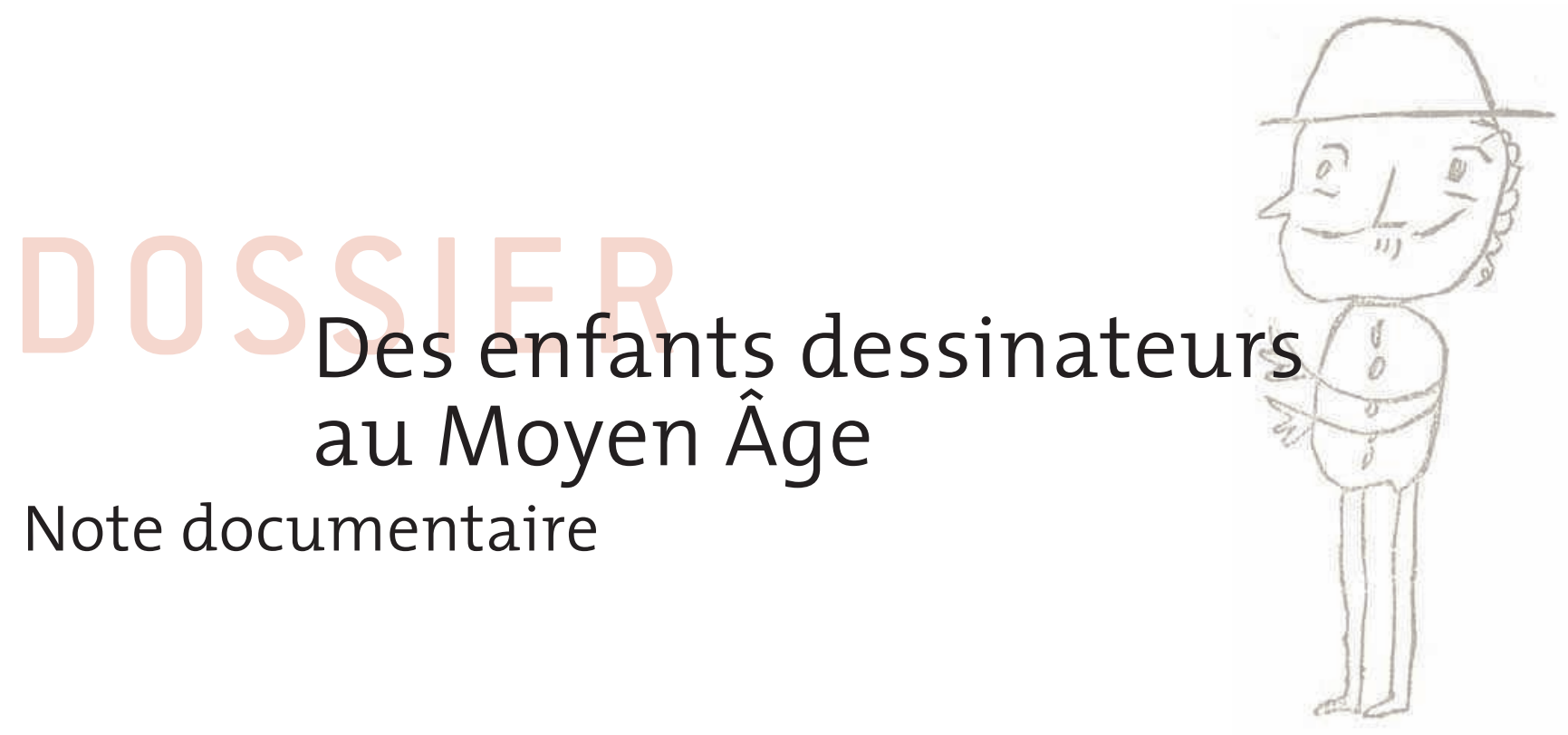

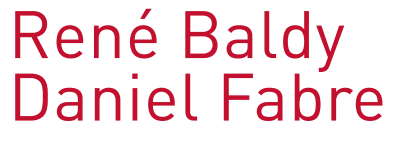

Depuis la publication des graffiti relevés sur les murs de Pompéi (Garucci 1856; Zangemeister 1875), la curiosité épigraphique et archéologique n'a cessé de découvrir des signes sur les parois, dont plusieurs ont été attribués à des enfants à partir d'un critère discutable : la maladresse dans l'exécution, c'est-à-dire l'ignorance du réalisme visuel. En fait, les cas où l'attribution enfantine ancienne est assurée sont très peu nombreux. Les plus riches se situent en Europe aux xiI ${ }^{\mathrm{e}}$ et xiII ${ }^{\mathrm{e}}$ siècles, et ce sont eux que nous avons choisi de présenter ici. Daniel Fabre a repéré et défini deux ensembles graphiques exceptionnels et proposé à René Baldy de les commenter du point de vue de la psychologie du dessin d'enfant appliquée à la figure humaine, esquissant, pour finir, les étapes de leur métamorphose en œuvres d'art dans les années récentes.

Novgorod 1230 : les dessins d'Onfim

Ancienne capitale de la Russie médiévale, Novgorod, au nord-ouest de Moscou, a fait l'objet, à la suite des dévastations de la Seconde Guerre mondiale, d'une fouille archéologique systématique dont un des résultats les plus frappants a été la découverte de centaines de textes rédigés sur des écorces de bouleau (Thompson 1967). Écrits exceptionnels ou ordinaires (contrats, lettres familières, lettres d'amour, comptabilités...), ces documents ont permis de reconstituer un univers graphique très dense dans une cité riche en monastères et en églises (Franklin 2002). Ces textes utilisent l'alphabet majuscule cyrillique et tendent à aligner les lettres non sur leur base, comme aujourd'hui, mais sur leur sommet. Au quinzième niveau de profondeur, datable des années 1224-1238, sont apparues des "pages d'écorce " consacrées à des exercices d'écriture et à des dessins. Sur certaines, par exemple à droite du cavalier du dessin 3, un nom est inscrit : Onfim. Nous avons de la main de ce garçon une douzaine de pages qui, le plus souvent, associent image et textes. Ces derniers légendent ou commentent les dessins ou bien énumèrent l'alphabet, comme c'est le cas pour la figure du cavalier à peine citée qui comporte dans son angle supérieur droit la suite incomplète des lettres, de $\mathrm{A}$ à $\mathrm{K}$. Le débat est ouvert sur le sens de ces alphabets : s'agit-il d'un simple 
exercice d'apprentissage, ou bien, comme le suggère la dispersion de cette forme de texte dans l'espace graphique de la ville, ont-ils un usage symbolique qui les apparenterait à des formules de protection ou de conjuration, l'alphabet étant la suite complète et maîtrisée du dicible, entre l'alpha et l'oméga (Franklin 2002 : 203, note 65) ? La figure humaine, associée à des silhouettes animales et à quelques objets prolongeant l'action du corps, est au centre de la production dessinée d'Onfim. Il est donc possible, d'une part, de déduire le moment du développement graphomoteur dont témoignent ces dessins et, d'autre part, de caractériser le style de ce jeune dessinateur dans ces tracés libres où il s'exprime à côté de la plus contrainte des écritures.

Les dessins d'Onfim présentent ce qu'avec Luquet (1927) on peut appeler une " narration graphique", c'est-à-dire le récit d'événements successifs, la description, séquentielle ou non, d'actions dramatiques. Tous les personnages dessinés sortent du même moule. Dans la généalogie que l'un de nous a décrite (Baldy 2008), il s'agit de bonshommes pseudo-têtards intermédiaires entre le bonhomme têtard et le bonhomme conventionnel filiforme. Deux éléments principaux nous conduisent à le caractériser ainsi. D'abord, on note que les bras du bonhomme ne sont pas rattachés à la tête, mais à la jonction de la tête et des deux traits verticaux ou à diverses hauteurs de ces traits, lesquels présentent dans leur partie supérieure un arrondi qui semble vouloir figurer le contour du tronc et qui se prolonge par deux segments rectilignes figurant les jambes. Bien qu'il manque un trait horizontal au niveau de la ceinture, l'ensemble ne donne pas l'impression d'un bonhomme sans tronc. L'arrondi du tronc et la longueur des traits verticaux figurant les jambes varient d'un dessin à l'autre, souvent en sens inverse : l'arrondi est absent dans les dessins 4 et 8 et sa courbure s'accentue dans les dessins 6 ou 10, alors que les jambes sont exagérément longues dans le dessin 6 et exagérément courtes dans le
Dessins 1 à 11

Les dessins d'Onfim, site archéologique de Novgorod, Russie, vers 1220
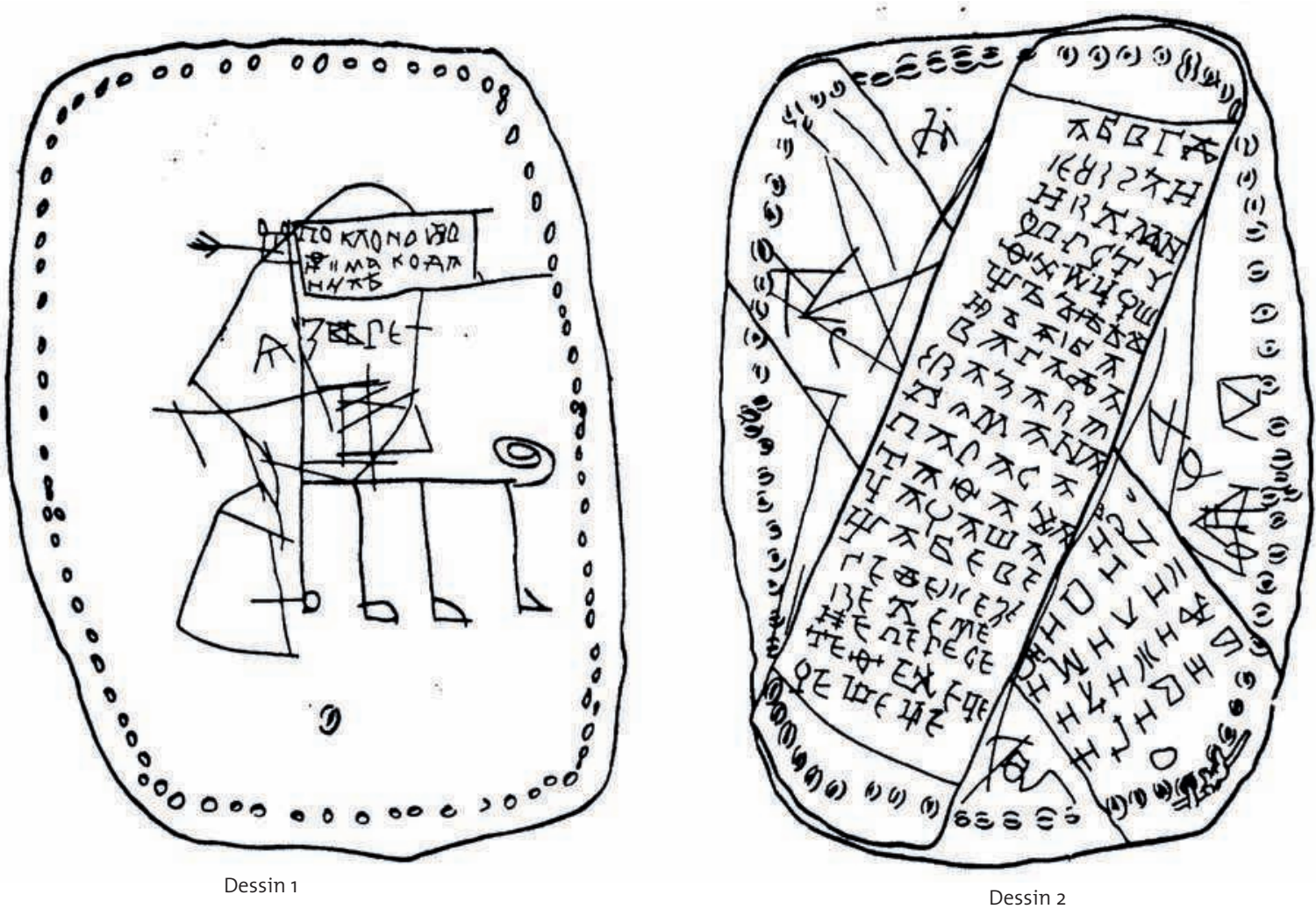


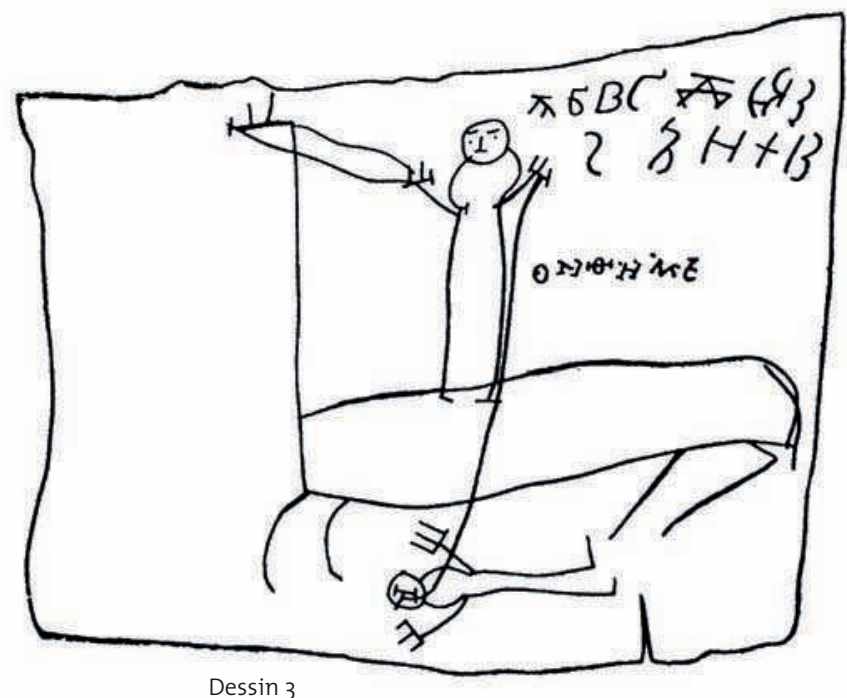

dessin 10. Les traits verticaux des jambes se terminent dans leur partie inférieure par un angle droit dont le côté horizontal mesure moins d'un centimètre et qui est lui-même délimité parfois par un tiret vertical de quelques millimètres, cet ensemble figurant les pieds du bonhomme. Les bras sont deux traits rectilignes tracés dans une orientation variable, depuis les bras levés en signe de victoire du dessin 4 aux bras ballants du dessin 10, en passant par la position en croix de certains bonshommes du dessin 5 et du bonhomme 6. Ces orientations des bras semblent déterminées par plusieurs facteurs : réponse à la nécessité de l'action du cavalier qui tient les rênes du cheval et qui soulève sa lance (dessin 3), expression d'une émotion telle que la joie de la victoire (4) ou la tristesse (10), souci d'éviter les superpositions de traits (5). Tous les bras se prolongent par des mains ou plus exactement, en l'absence de paume, par des doigts "en râteau " (Baldy 2008: 65). Selon le dessin, le nombre de doigts varie de trois (dessin 7) à sept ou huit dans le dessin 4, où Onfim se représente à côté de son père, précise sa légende, attribuant à celui-ci plus du double de ses doigts. Tous les bonshommes sont dessinés de face, même les cavaliers, dans une position verticale ou horizontale. Les visages sont relativement soignés. Les yeux surmontés de sourcils, le nez et la bouche sont généralement indiqués par de simples traits, mais parfois de façon plus sophistiquée, ainsi les yeux cerclés du bonhomme 10. Le bonhomme 6 semble posséder deux oreilles et peut-être quelques cheveux.
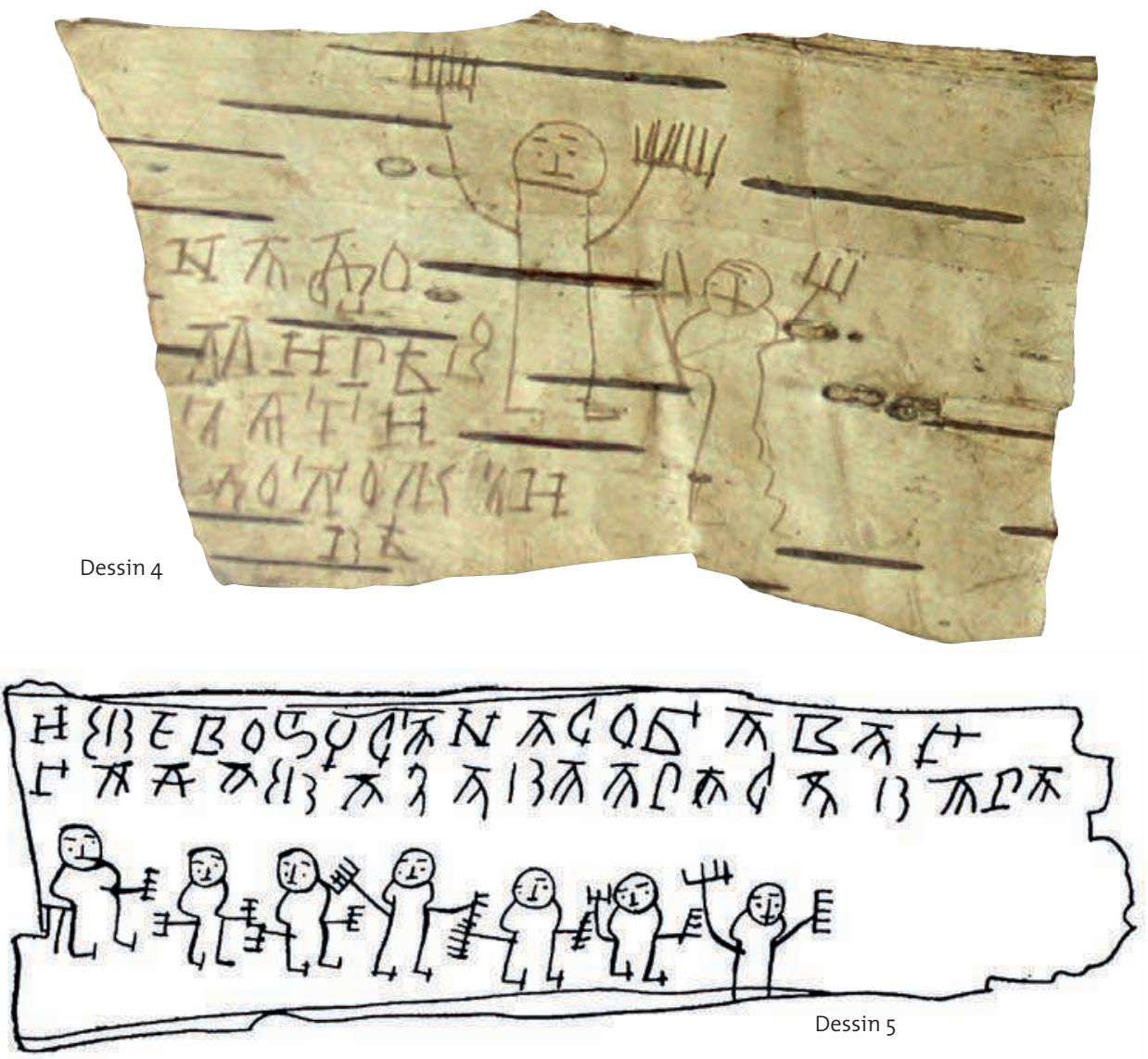
D'une façon générale, les signifiants graphiques qui composent les dessins, et notamment les bonshommes, semblent avoir été puisés dans un répertoire fini, de même que les signes de l'écriture qui les accompagne.

À côté des bonshommes, Onfim aime à représenter des scènes de bataille avec personnages à cheval. On en compte cinq - 2, 3, 7, 8 et 9-, les scènes 3,7 et 8 étant les plus explicites; le $n^{\circ} 3$ peut être lu comme un cavalier sur son animal, tenant les rênes d'une main et de l'autre tuant d'un coup de lance un adversaire allongé sur le sol. Ces dessins présentent toutes les caractéristiques de la phase du réalisme intellectuel :

- mélange de points de vue : cavalier de face et animal de profil;

- superposition du cavalier debout et de sa monture;

- déplacement latéral des pattes situées du côté opposé à l'observateur pour les rendre visibles; notons cependant que la forme angulaire des pattes arrière confère un certain dynamisme à l'animal, qui semble se préparer à bondir ;

- non-respect des proportions : allongement du cheval du dessin 7 pour qu'il supporte deux cavaliers, allongement de la lance du dessin 3 pour qu'elle atteigne l'ennemi sans quitter la main du cavalier;

- absence de ligne de base sur laquelle reposeraient les éléments dessinés (c'est, rappelons-le, la règle pour l'écriture à Novgorod à cette époque);

- absence de superpositions (bras des sept bonshommes alignés du dessin $\left.n^{\circ} 6\right)$;

- présence de légendes écrites.

Dans certaines scènes $(7,8,9)$, Onfim a dessiné plusieurs cavaliers qui semblent se situer sur des plans plus ou moins éloignés du spectateur, suggérant l'idée de perspective. Il est possible que le dessin 8 présente trois moments successifs d'une narration graphique (Luquet 1927). Le cavalier situé à droite de la scène est petit parce qu'il arrive et se trouve encore loin du spectateur; il est ensuite au premier plan, au centre de la scène, tuant un ennemi, puis il s'éloigne vers la gauche.

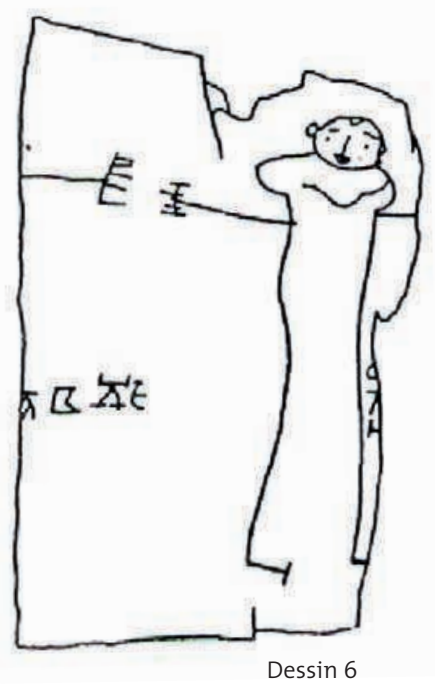

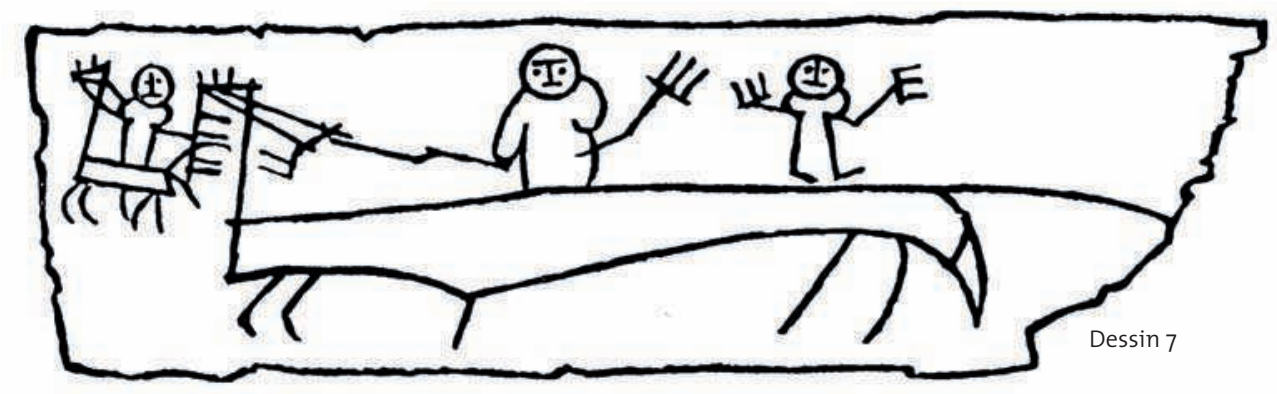

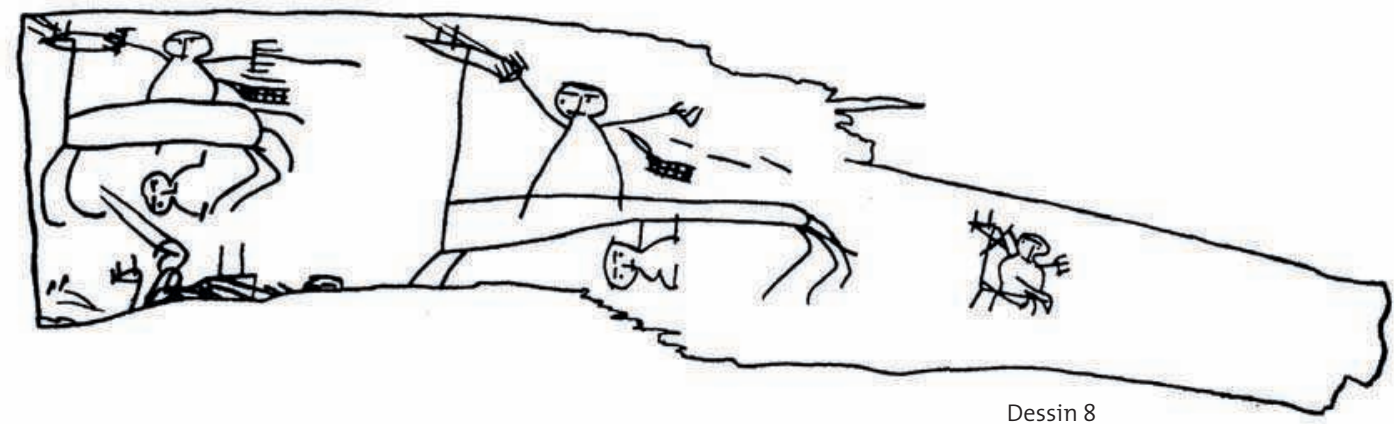



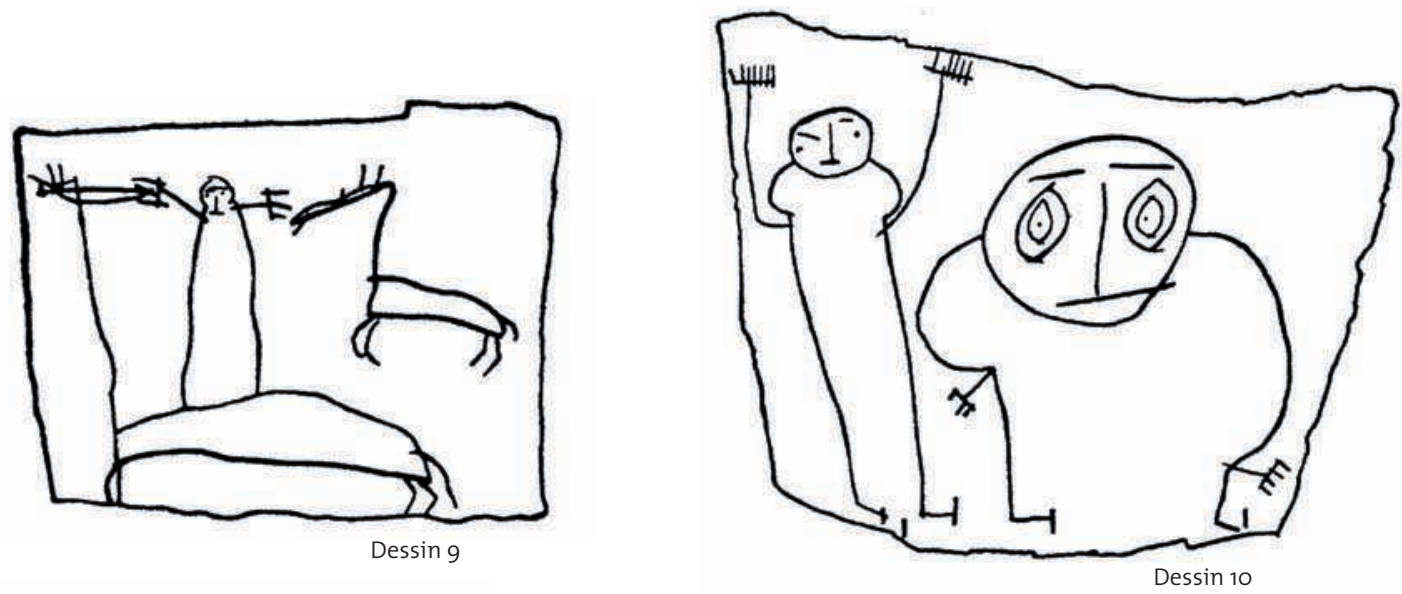

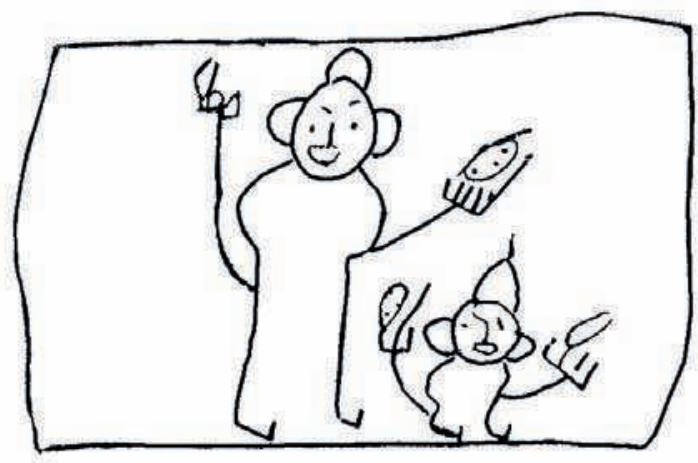

Dessin 11

Onfim a parfois tenté de figurer une manifestation affective avec des moyens expressifs assez complexes. Les dessins 4, 10 et 11 présentent deux personnages. Dans le dessin 10, les deux bonshommes, bien que conformes au schéma habituel, sont très différents l'un de l'autre, au point que l'on peut penser que le dessinateur a voulu cette différence pour exprimer une émotion. Le personnage du second plan, identique aux autres, lève les bras pour manifester sa joie. Le bonhomme du premier plan, en revanche, exprime un profond abattement: les deux yeux écarquillés, figurés par deux cercles concentriques formant des couronnes surmontées d'un trait horizontal, les sourcils et la bouche, figurée de même, confèrent au visage une tristesse résignée. Si cette émotion a été intentionnellement marquée, elle témoigne d'une grande maturité (Brechet, Picard et Baldy 2007; Picard, Brechet et Baldy 2007). Le tronc en boule, dissymétrique et exagérément arrondi pour contenir le «cœur gros ", accentue encore cet effet d'abattement. Les tout petits bras tombant attachés à la limite inférieure de l'arrondi du tronc, les petites mains avec peu de doigts, et les jambes, minuscules si on les compare à celle du bonhomme 6 , soulignent également l'impuissance du personnage. Dans le dessin 11, les deux personnages, à grandes oreilles, sont coiffés d'un casque (?) et tiennent des objets (peut-être des sabres) dans leurs deux mains. Ils se distinguent essentiellement par la taille, ce qui laisse penser que le dessin représente un adulte et un enfant. Peut-être Onfim a-t-il voulu se représenter à côté de son père?

Que dire des capacités graphomotrices de ce jeune dessinateur? D'abord, tout porte à penser qu'Onfim était droitier. Le dessin 5 est composé de sept personnages debout, de même taille, alignés, de face, très probablement dessinés de gauche à droite. L'ajustement des bras des bonshommes laisse penser que le dessinateur a d'abord tracé le bonhomme de gauche et qu'il a continué vers la droite en dessinant les bras des bonshommes successifs en quinconce (les trois premiers bonshommes en partant de la gauche) ou en ajustant leur orientation (les quatre bonshommes suivants). On observe que le changement de l'orientation entraîne un allongement de leur longueur. De façon récurrente, les caractères du dessin d'Onfim confirment cette hypothèse : l'angle des pieds est orienté vers la droite, leur partie horizontale est donc tracée de gauche à droite, ce qui est le sens préféré des droitiers; de même, c'est toujours le profil gauche de l'animal qui est représenté.

Quant au stade de développement dont témoignent ces dessins, l'évaluation de ces bonshommes selon le barème proposé par Goodenough (1926) situerait Onfim entre quatre et cinq ans. Cet âge correspond bien au début de la phase 
du réalisme intellectuel (de cinq à sept ans environ) qu'illustrent les scènes de bataille. Cependant, plusieurs facteurs incitent à penser qu'Onfim était un peu plus âgé. Il ne dessine plus un bonhomme isolé, mais des scènes plus ou moins complexes. Il est très probable qu'il a fait ces dessins librement, sans consigne de la part d'un adulte. Or, on sait que lorsque le bonhomme n'est qu'un élément d'une scène et que, lorsque le dessin est un «dessin libre », les personnages sont moins sophistiqués ou moins soignés que lorsqu'un adulte demande expressément à l'enfant: "Dessine-moi un bonhomme", comme c'est le cas dans le test de Goodenough. De plus, si notre interprétation du dessin 10 est correcte, on peut penser que la modulation des signifiants graphiques pour marquer l'émotion ressentie par le personnage témoigne du niveau de développement intellectuel et affectif d'un enfant de six ou sept ans. Il faut aussi tenir compte de l'effet Flyn, du nom du chercheur qui a mis en évidence l'augmentation régulière, dans l'histoire occidentale, du niveau intellectuel des enfants tel que le mesurent les tests. Ce changement a des causes multiples : scolarité, pratiques éducatives familiales, amélioration des conditions de vie, évolution technologique de la société, etc. Si nous n'en tenons pas compte, il nous conduit à surestimer les capacités cognitives et donc graphiques d'Onfim. À âge égal, un enfant $\mathrm{du} \mathrm{xx}^{\mathrm{e}}$ siècle (qui est la norme de Goodenough) anticipe probablement sur ce que faisait un enfant du xiII ${ }^{\mathrm{e}}$ siècle. Ainsi, les dessins d'Onfim correspondent aux dessins que ferait aujourd'hui l'enfant moyen de cinq ans, mais il est probable que l'enfant moyen de cinq ans vivant au xiII ${ }^{\mathrm{e}}$ siècle en Russie dessinait moins bien que l'enfant de cinq ans d'aujourd'hui et que les dessins retrouvés par les archéologues ont été faits par un garçon un peu plus âgé. De plus ces dessins n'ont pas été exécutés avec un crayon sur du papier de qualité mais gravés avec un stylet sur de l'écorce de bouleau, ce qui est nettement moins commode.
Fig. 1 Anthropomorphes du réseau Vidal-Jullia, Trauc del Calèl, Tarn.

\section{Sorèze, vers 1150 :}

les dessins des petits mineurs

Le contexte de création des dessins de Sorèze est sensiblement différent. Nous sommes dans le sud de la France, sur le versant septentrional de la montagne Noire (département du Tarn), où se trouve un petit plateau karstique dénommé causse de Sorèze, du nom de la petite ville toute proche. Un système très complexe de grottes et de galeries a été creusé dans la colline calcaire par les eaux d'infiltration, qui forment une rivière souterraine à 100 mètres de profondeur. Les premiers textes faisant état de ces merveilles souterraines datent du milieu du xvII ${ }^{\mathrm{e}}$ siècle, ils confirment le toponyme local attesté en 1508 mais évidemment antérieur: le Trauc del calèl, le "gouffre de la lampe». L'exploration systématique s'est déroulée à partir de la fin du xviII siècle et est aujourd'hui l'œuvre des spéléologues. En avril 1973,

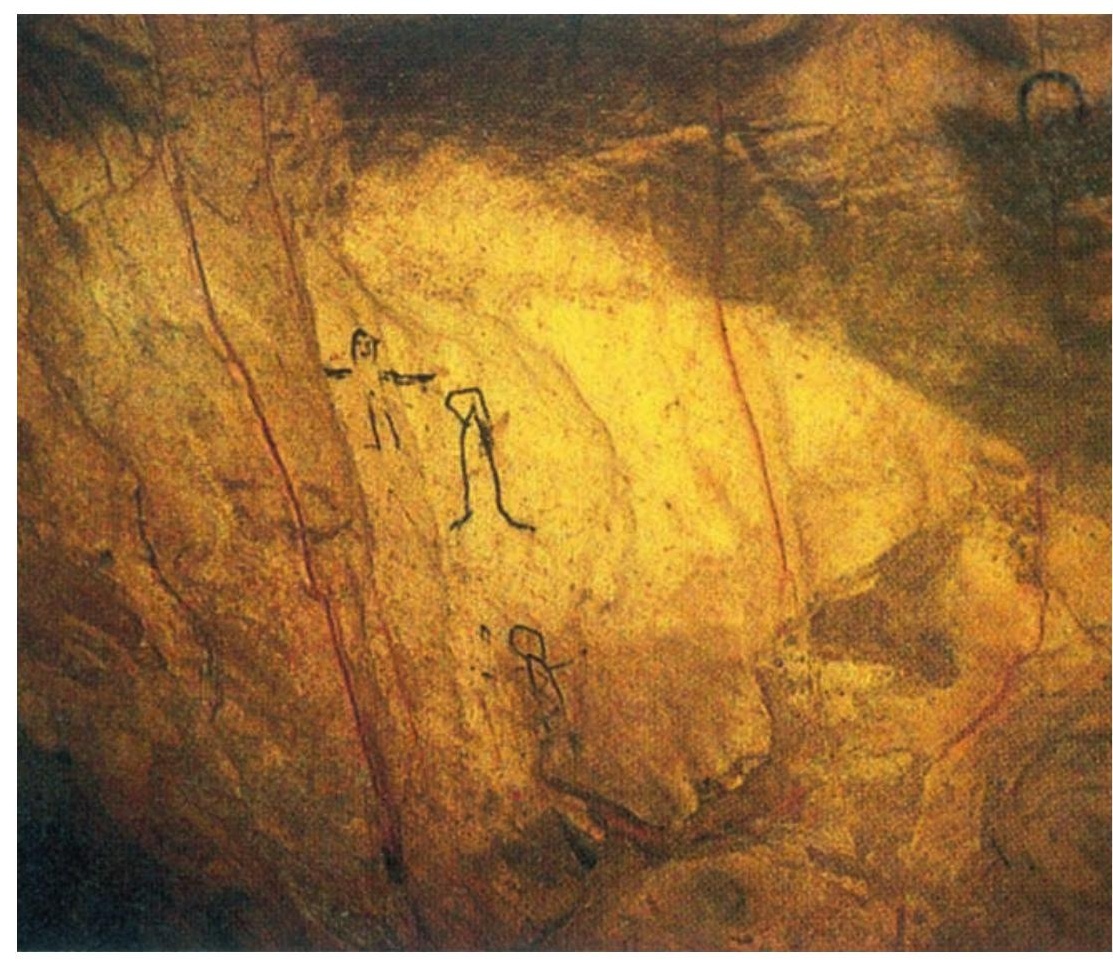


Fig. 2 Dans la grotte du Calèl. Empreintes des torches fixées dans l'argile par les enfants. Les dessins ont été tracés par la pointe charbonneuse de ces torches.

Dessins 12 à 22

Les enfants de la mine, Sorèze, Languedoc (site archéologique de la grotte du Calèl), vers 1220 Relevés de Claire Marin d'après des photographies de Lucien Gratté.
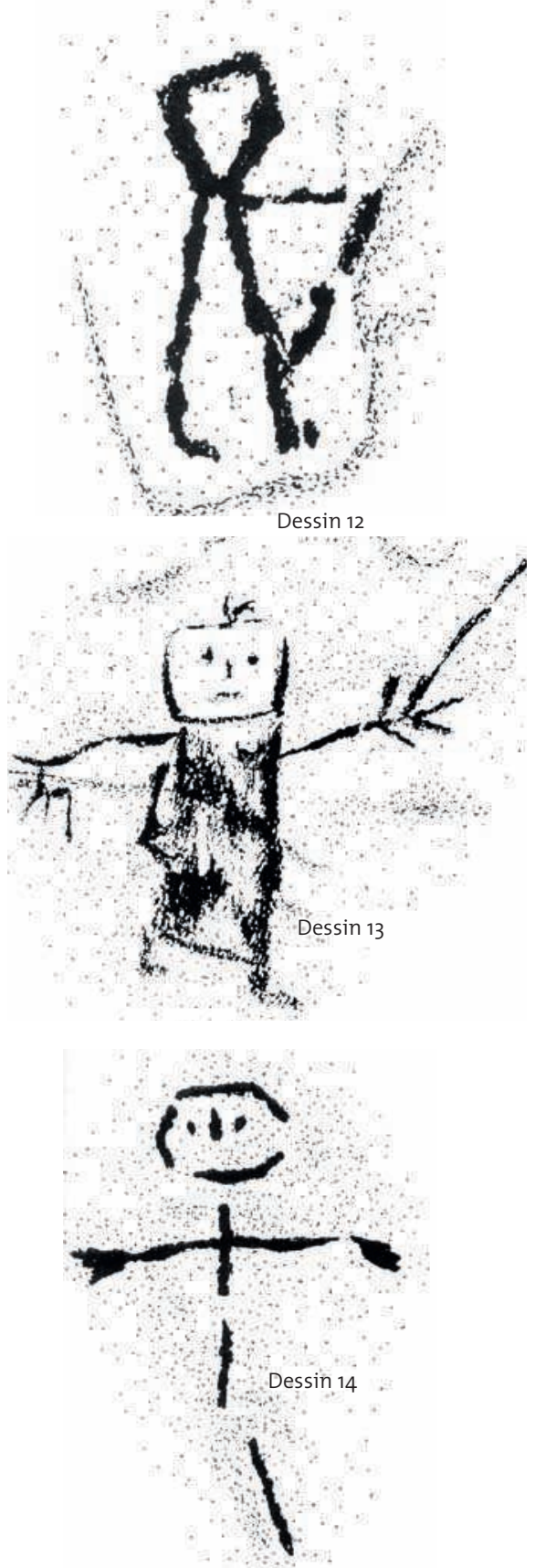

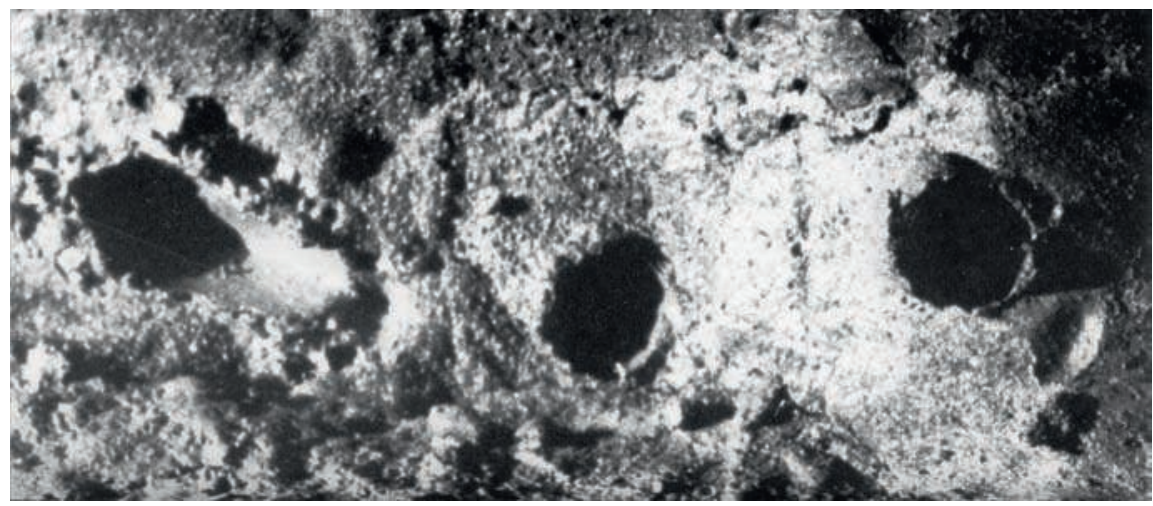

ceux-ci explorent une partie du réseau dit Vidal-Jullia, du nom de ses premiers découvreurs. Ils se trouvent devant l'évidence d'une exploitation ancienne de la grotte : des galeries aménagées, des ponts, des escaliers, des tessons de poterie, des marques de torches, des traces régulières d'outils métalliques sur les murs... Le fait peut-être le plus troublant est la présence d'empreintes calcifiées dans l'argile, surtout des pieds d'enfants, ce qui correspond bien à la hauteur réduite des galeries creusées ou élargies dans l'épaisseur rocheuse. L'hypothèse qu'il y ait eu là un site d'extraction dont les mineurs étaient principalement des petits garçons - situation fréquente en Europe jusqu'au milieu du xix ${ }^{\mathrm{e}}$ siècle et encore attestée aujourd'hui ailleurs dans le monde - s'est très vite imposée. Devant l'énorme quantité d'argile extraite et ramenée en surface, on a d'abord supposé que ces mineurs travaillaient pour des potiers. Cependant, des analyses plus récentes ont démontré que c'est en fait un minerai de fer, des nodules pris dans l'argile, très pauvres en métal, qui a été extrait du Calèl entre le xi ${ }^{\mathrm{e}}$ siècle et le $\mathrm{XII}^{\mathrm{e}}$ siècle et fondu sur place, en quelques points du plateau. Il semble, d'après les datations au carbone 14, que cette exploitation ait été fermée vers 1150. Sur les parois, des traces de gravures géométriques se laissent entrevoir, mais la stupéfaction des premiers explorateurs fut à son comble lorsqu'ils découvrirent, en deux endroits voisins, des figures au contour noir, visiblement tracées au charbon de bois à l'aide de la pointe des torches. Le style de ces dessins, dans ce contexte particulier, les a fait logiquement attribuer à des enfants. Onze images au total, qui représentent toutes des figures humaines, avec ou sans visage, dont cinq évoquent, plus ou moins nettement, la tenue et les gestes du travail minier - nous y reviendrons. Un fait surprend cependant: ces figures en groupe sont nettement détachées l'une de l'autre et vierges de superposition postérieure; de toute évidence, nous ne sommes pas devant des panneaux de graffiti où le tracé appelle le tracé, il semble même que ces dessins aient été faits puis enfouis là d'un geste délibéré qui signait la fin définitive de la mine. L'un des premiers commentateurs de cet ensemble pariétal, Lucien Gratté, émet l'hypothèse perspicace selon laquelle la mine a été fermée sur ordre du comte de Toulouse - le blason portant la croix du Languedoc est sculpté en relief près de l'entrée et un édit de 1223 interdit l'exploitation du monde souterrain dans le comté pour des raisons religieuses -, d'où la première datation proposée pour ces dessins (Gratté 1988 : 45). L'analyse géologique et les datations invitent aujourd'hui à anticiper au moins un demi-siècle la fermeture définitive de la mine, mais le débat sur ce point n'est pas clos. 

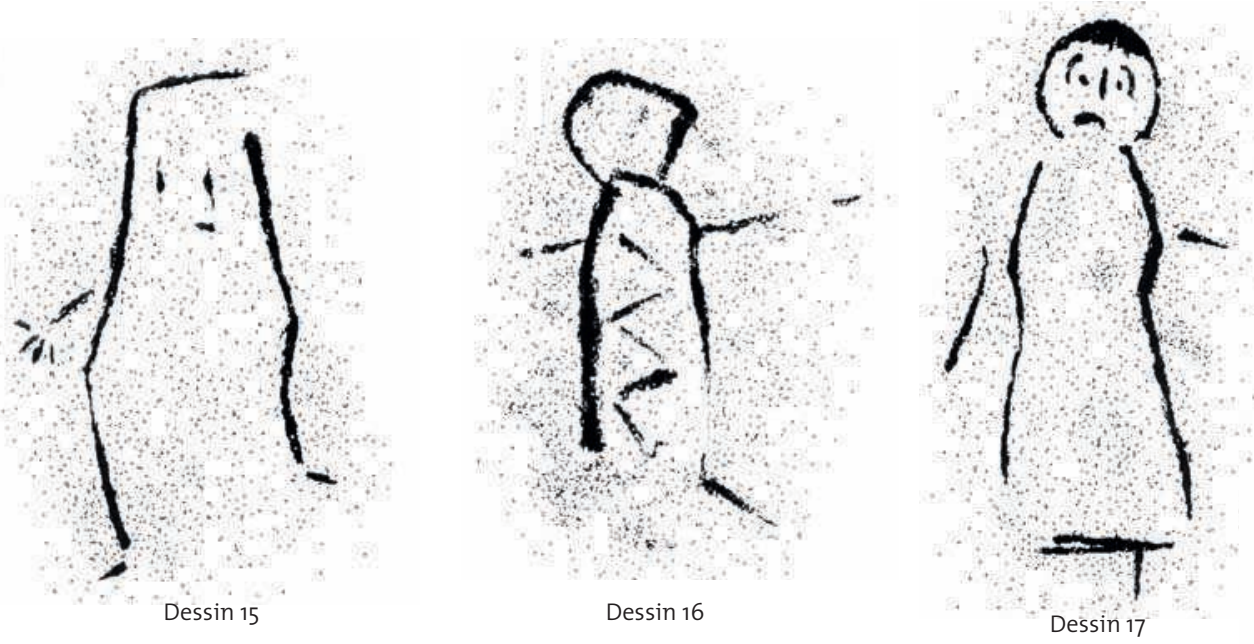

Cette reconstitution du contexte de création des dessins permet surtout de comprendre un fait très singulier qui, jusqu'à présent, n'a pas retenu l'attention des commentateurs. À la différence des dessins d'Onfim, nous n'avons pas affaire ici à une main unique, mais plutôt à un groupe d'enfants ouvriers assez homogène dont tout porte à penser qu'ils n'étaient pas scolarisés, aucune lettre, gravée ou dessinée, n'accompagnant ces dessins. C'est ce qui explique sans doute l'hétérogénéité des solutions et, pourrait-on dire, des styles graphiques présents sur les parois de la mine. À cela s'ajoute le fait que chaque enfant a conçu son dessin comme une signature, ici indissociable d'un autoportrait. Ceux qui possédaient une main plus sûre, les plus âgés du groupe, ont tenté de se conformer à un certain réalisme visuel; soit en représentant l'équipement de l'ouvrier - les dessins 15, 16, 17, 20 et 22 figurent une sorte de longue robe, les dessins 12 et 21 fixent le geste caractéristique par lequel le mineur attaque la paroi à l'aide de son pic-, le dessin 12 le fait par un profil très schématique, le dessin 21 de façon très précise, au point que l'on peut y reconnaître l'œuvre d'un plus grand, peutêtre le chef de l'équipe. Les autres figures sont des bonshommes enfantins, de face, qui présentent donc le paradoxe de renvoyer aux mêmes stades graphomoteurs tout en choisissant des solutions graphiques assez sensiblement différentes. Arrêtons-nous aux caractéristiques les plus remarquables :

Les dessins 16 et 20 sont des bonshommes têtards très sommaires. En revanche, le dessin 12 est un pseudo-têtard de profil, très rare aujourd'hui, orienté vers la droite (on devine le nez), avec deux bras en avant dont les extrémités sans mains se rejoignent comme pour tenir le pic à l'aide duquel il attaque la paroi.

Le dessin 13 a semble-t-il été exécuté selon la procédure du bonhomme têtard vu de face (tête et deux traits verticaux), mais le bas du tronc a été délimité par un trait horizontal, après quoi le tronc a été noirci. Deux jambes très courtes se terminent par des pieds de profil orientés vers la droite. Les bras filiformes sont munis de doigts en "arêtes de poisson ». Il semble que le bonhomme tienne un objet en forme de bâton (un outil) dans la main gauche. Soulignons que cela ne signifie pas que le dessinateur ait voulu dessiner un bonhomme gaucher. Il est plus probable que le dessinateur, étant droitier (ce qui est étayé par les pieds orientés vers la droite), ait dessiné le bâton dans la main du bonhomme qui est face à sa main droite (voir Baldy 2008 : 148). De plus, d'un point de vue strictement moteur, il est difficile pour un droitier de dessiner l'objet dans la main droite du bonhomme, car ce choix l'oblige à couvrir de la main la partie du dessin déjà tracée.

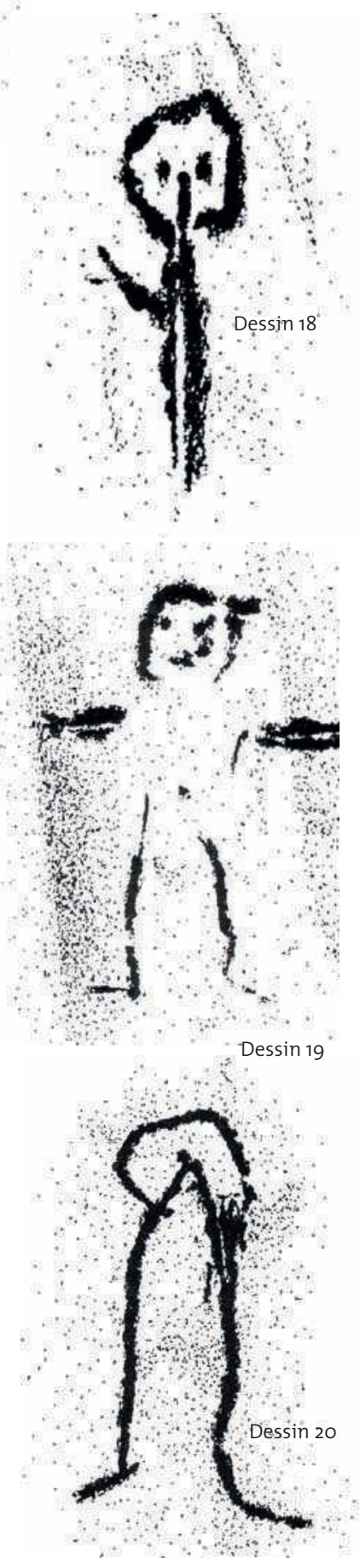



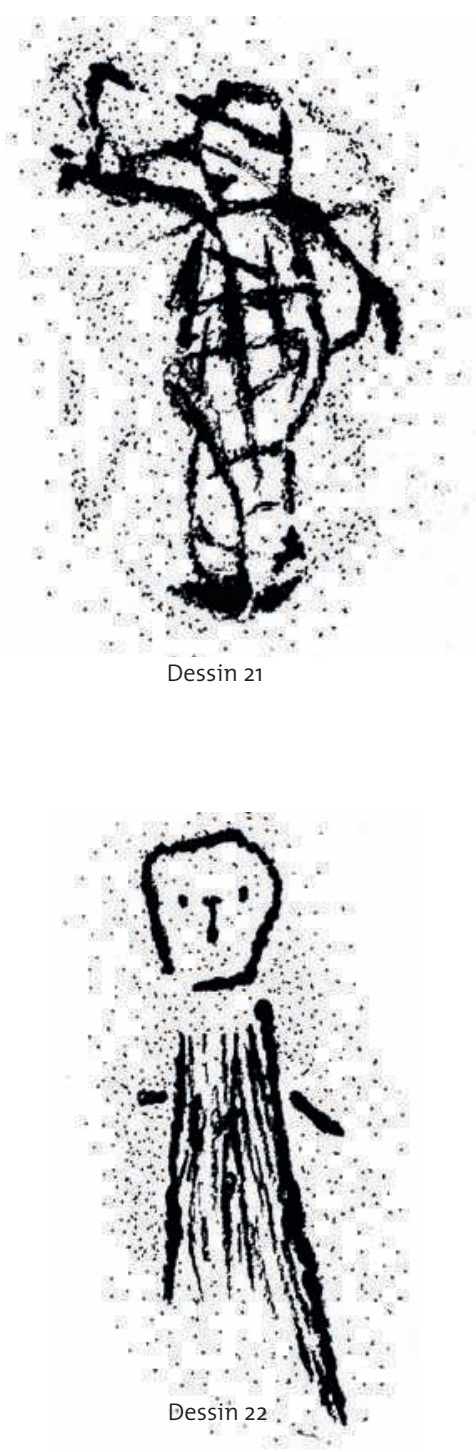

Le dessin 14 (filiforme unijambiste) correspond à un dessin stylisé. C'est presque un pictogramme. Le bonhomme est unijambiste et n'a pas de bouche.

Le dessin 15 est d'une formule "fantôme" plus originale qui rappelle le Jimi contour (voir ci-dessus p. 139). On observe que le bonhomme n'a pas de nez, si l'on admet que le trait horizontal figure la bouche et n'a qu'un bras terminé par quatre doigts en éventail. On a beaucoup d'exemples de bonshommes manchots, dont seul le bras utile a été dessiné. Le tronc du bonhomme n'est pas bien délimité. La limite «virtuelle» entre le tronc et les jambes pourrait se situer aux points d'inflexion des deux lignes verticales. Il manque la ceinture. Le dessin semble avoir été exécuté en deux traits tracés avec une assurance qui témoigne d'un bon contrôle moteur et donc d'une certaine habitude des activités graphiques. Le dessinateur a probablement commencé son tracé au point le plus haut et exécuté d'un trait le côté gauche du dessin, selon le schéma habituel, il l'a ensuite complété en traçant d'un trait le côté droit de bas en haut. Les yeux, la bouche, le bras et les doigts ainsi que les pieds "ouverts » ont sans doute été ajoutés par la suite.

Le dessin 17 est plus expressif que les autres. Les yeux écarquillés entourés de cernes, le trait de la bouche incurvé vers le bas expriment la tristesse du visage. Le haut de la tête est noirci pour figurer la chevelure ou un casque de mineur. La forme du tronc arrondi à la poitrine et en forme de robe suggère, peut-être, un personnage féminin ou, aussi bien, le sarrau resserré à la taille que devaient porter les petits mineurs. Notons que l'assurance du tracé n'est pas sans rappeler celle de l'auteur du dessin 15.

Le dessin 18 , très sommaire, possède un corps très étroit et est manchot.

Le dessin 19 est original par l'absence du contour du tronc, symbolisé toutefois par le nombril, que l'on peut interpréter comme un signe d'immaturité. Sur ce seul dessin, les bras sont figurés par un double trait, en tube.

Le dessin 22 est plus sommaire. C'est un pseudo-têtard avec les bras rattachés aux jambes. Le contour de la tête, probablement tracé à partir du bas en tournant dans le sens des aiguilles d'une montre, est mal fermé. Il n'a pas de bouche. L'espace situé entre les deux traits figurant les jambes est noirci par des traits verticaux qui indiquent, peut-être, le sarrau que portait le mineur.

Quel âge avaient les travailleurs enfants qui ont abandonné la mine en y laissant ces signatures graphiques? On peut, comme pour Onfim, appliquer à leurs dessins le barème de Goodenough :

Si ces dessins avaient été exécutés au xx siècle, le barème de Goodenough permettrait d'attribuer aux dessinateurs des âges allant de trois à six ans. Ici encore, compte tenu de l'effet Flyn et de ce que l'archéologie nous apprend des conditions d'exécution des dessins, il est certain que les enfants qui les ont faits sont plus âgés. Il n'est pas impossible que certains dessins aient été exécutés par un grand adolescent ou un adulte. C'est probable pour le dessin 21, le plus chargé en détails visuels, et pour le dessin 14, qui correspond au dessin stylisé du bonhomme tracé d'un trait rapide par un adulte d'aujourd'hui.

\section{Documents, monuments, œuvres}

Sans proposer ici une chronique exhaustive de la réception de ces dessins enfantins venus du Moyen Âge, il est instructif d'esquisser la trajectoire qui va de leur découverte à leur reconnaissance internationale, d'autant que leur destin contemporain est très similaire. 
Au commencement est l'archéologie; la révélation de ces figures s'insère dans un ensemble plus vaste - à Novgorod, une ville médiévale tout entière avec ses écrits, à Sorèze, une ancienne mine que l'on a prise, dans un premier temps, pour une simple carrière d'argile. Pour les chercheurs qui furent les premiers à découvrir et à décrire ces images, elles constituent des documents un peu particuliers dans la mesure où elles représentent des personnages et des scènes inaccessibles à la saisie archéologique. L'apprentissage scolaire d'Onfim ou les scènes de bataille qu'il dessine enrichissent directement, de visu, la connaissance des pratiques anciennes. Les costumes des mineurs de Sorèze, où certains crurent un temps reconnaitre les moines de l'abbaye voisine, confirment l'iconographie rare et tardive des métiers d'extraction, laquelle explique même le toponyme - lo Trauc del calèl - par l'utilisation, à côté de la torche rudimentaire, de lampes à huile en métal (calèl en occitan) dont il semble que certains mineurs les portaient déjà fixées au front, ainsi que peut le suggérer le dessin 21. Sans attacher une importance particulière à une archéologie de l'enfance qui ne prendra son autonomie qu'à une date très récente (Baxter 2008), et alors même que Novgorod et Sorèze laissent entrevoir les deux destins contrastés qui attendaient les très jeunes garçons à cette époque - l'étude et le jeu, d'une part, le dur travail physique, de l'autre-, les inventeurs des deux sites saluent la nouveauté documentaire de leurs trouvailles, ils en publient scrupuleusement les résultats, mais pas plus. Alors même qu'entre 1988 et 1995 des archéologues professionnels approfondissent la fouille du site de Sorèze, l'événement ne sort pas, pendant des années, des inventaires spécialisés à usage savant.

La situation évolue lorsque l'ensemble dans lequel ces documents se trouvent insérés fait l'objet d'un classement officiel qui lui confère la qualité de site protégé et de monument historique. La décision est précoce pour Novgorod, à cause de son statut d'ancienne capitale. Elle est plus lente pour Sorèze, ce qui rend le cas particulièrement intéressant ${ }^{1}$. Les découvreurs ont conscience qu'ils ont mis au jour des ensembles exceptionnels et que la présence de ces dessins, dont la datation ne fait guère de doute puisque les galeries ont été obstruées volontairement et sont de ce fait inaccessibles depuis le Moyen Âge, est un argument de poids pour obtenir le classement du site. De fait, il apparaît très vite que ces dessins distinguent la mine du Calèl de toutes les autres exploitations minières présentes sur les deux versants de la montagne Noire depuis la protohistoire et, surtout, depuis l'époque romaine. Une dizaine d'années après sa découverte (arrêté du 10 octobre 1977), le site est donc protégé à des fins d'étude. Plus tard, après de nombreuses protestations contre l'incurie des autorités locales, il est inscrit sur la liste supplémentaire des Monuments historiques sur proposition de la commission régionale de Midi-Pyrénées ( 2 avril 1991). Enfin, les partisans d'une "sanctuarisation" définitive obtiennent le classement comme monument historique (décret du 8 octobre 1995). Quelle place tiennent les dessins des petits mineurs dans cette consécration? De fait, elle reste floue et ambiguë. Ainsi, dans sa défense très argumentée de la sauvegarde, le Comité international d'art rupestre (Rouzaud, Mauduit et Calvet 1994) élimine la moindre allusion à l'âge des dessinateurs. Reportant son attention vers les preuves désormais bien établies de l'exploitation minière, il propose des dessins une lecture fonctionnelle : ceux-ci marqueraient essentiellement la topographie de la mine, signaleraient éventuellement des dangers particuliers, baliseraient ce que les archéologues nomment les "axes logistiques» du circuit souterrain et, enfin, représenteraient les mineurs en tenue et en situa-

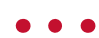

1. Nous remercions JeanDaniel Pariset, directeur de la Médiathèque du Patrimoine, de nous avoir orienté dans le maquis des archives qui enregistrent le classement de la mine du Calèl. 
tion de travail. Quant à la qualification d'«art ", elle est utilisée par convention pure : toute trace intentionnelle sur la paroi est dite "art pariétal", sans que jamais rien ne soit tenté pour esquisser une description précise des moyens expressifs maîtrisés par les dessinateurs. La demande de classement définitif s'appuie d'abord sur l'unité que forme la mine et les traces d'un traitement du minerai en surface, les images venant comme un supplément qui vaut pour sa grande rareté. La monumentalisation - qui prévoit la possibilité de visites touristiques - se nourrit du document, un point c'est tout.

Ce n'est qu'à une date très récente que le monde de l'art accueille ces images enfantines médiévales. L'historien de Novgorod Valentin Yanine, qui a consacré un demi-siècle à l'étude du système social de la ville, insère systématiquement Onfim et ses dessins dans les articles de synthèse qu'il publie en anglais aux États-Unis. La reprise de ces informations dans des revues sur l'art médiéval, d'une part, et l'éducation artistique, de l'autre, et ce dès 1985 , déclenchent un processus de reconnaissance très rapide où se rencontrent tous les acteurs de l'actuelle renaissance d'intérêt pour l'art enfantin (Ianine 1990; 1997; Yanine 1964; 1985). C'est cependant la grande exposition "When We Were Young ", organisée à la fin de 2006 au Krannert Art Museum (Urbana, Illinois) par l'historien d'art Jonathan Fineberg, qui fait entrer Onfim dans la galerie des enfants dessinateurs reconnus, dont il inaugure une histoire qui court désormais du Novgorod médiéval jusqu'aux collections de Gabriele Münter, Wassily Kandinsky et Jean Dubuffet. On ne se demande même plus si Onfim est à proprement parler un artiste, il lui suffit d'être un enfant et donc nécessairement un créateur dans ce contexte de célébration de «l'œil innocent » et de la créativité enfantine. En Russie, des cercles consacrés à la formation des enfants au dessin et à la peinture portent aujourd'hui son nom, l'histoire reconstruite de sa vie connaît un succès mondial, on trouve aujourd'hui plus de trois millions de références à l'écolier de Novgorod sur Internet. Dans le cas des bonshommes du Calèl, leur découverte et leur artification sont le fruit récent d'un pur hasard auquel l'un d'entre nous fut mêlé sans le savoir. En décembre 2006, un blog très documenté consacré à l'art brut, "Animula vagula ", rapporte que son auteur, poussée par la pluie à se réfugier dans le musée du quai Branly, a découvert inopinément dans la salle de cinéma un séminaire assorti de projections qui présentaient cette trouvaille en laquelle elle reconnut aussitôt des œuvres originales qu'elle célèbre à sa manière :

Je me suis glissée au premier rang des étudiants et j'ai regardé le diaporama que commentait à ce moment un conférencier aux accents occitans. Et alors là, le choc, mes petits animuliens! Ce que je voyais, cétait du brut et je le connaissais pas : des dessins au charbon laissés par des enfants-travailleurs dans une mine d'argile bouchée dès le début du xiII ${ }^{\mathrm{e}}$ siècle. C'est à Sorèze, au sud de Castres, nous a dit le prof qui ressuscite ces trésors. [...] Laissez-moi vous dire que j'ai eu plaisir à me retrouver dans la peau d'une étudiante. J'ai pu vérifier la chose suivante : avec l'art brut même le passé a de l'avenir.

On comprend la surprise de l'amateur d'art marginal : ces figures aux yeux grands ouverts sur la nuit souterraine lui évoquent les apparitions dont regorgent les dessins d'enfants et dont Henri Michaux ou Jephan de Villiers ont donné des versions sidérantes. Nul doute, étant donné l'économie formelle et la surprenante diversité de ces images, qu'elles ne possèdent tous les caractères d'une " primitivité » que, sans y prétendre, cette note va contribuer à faire reconnaître. 


\section{Bibliographie}

\section{BALDY, René}

2008 Dessine-moi un bonhomme.

Dessins d'enfants et développement cognitif.

Paris, Inpress éditions.

\section{BAXTER, Jane Eva}

2008 "The Archaeology of Childhood", Annual Review of Anthropology 37 : 159-249.

Brechet, Claire, PiCARD, Delphine, et BALDY, René

2007 « Expression des émotions dans le dessin d'un homme chez l'enfant de 5 à 11 ans./

Expression of Emotions in the Drawing of a Man in the 5 to 11-year-old Child 》, Canadian Journal of Experimental Psychology //Revue canadienne de psychologie expérimentale 61(2), June : 142-153.

FINEBERG, Jonathan

2006 When We Were Young. New Perspectives on the Art of the Child. Krannert Art Museum, University of Illinois at Urbana, Oct.-Dec. 2006, University of California Press.

\section{FRANKLIN, Simon}

2002 Writing, Society and Culture in Early Rus, c. 950-1300. Cambridge, Cambridge University Press.
GARRUCI, Raphael

1856 Graffiti de Pompei. Paris, Benjamin Duprat, libraire de l'Institut.

\section{GoOdEnOUGH, Florence}

1926 L'Intelligence d'après le dessin, le test du

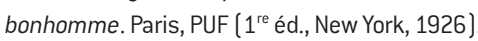

\section{Gratté, Lucien}

1985 Survivance de l'art pariétal. Millau, Impr. Maury.

1988 Chroniques d'une caverne en Languedoc. Le Traouc del calel à Sorèze. Toulouse, Spelunca.

\section{IANINE (OU YANINE), Valentin Lavrentievich}

1964 "The Dig at Novgorod", Readings in Russian Civilization I, Thomas Riha ed. Chicago, University of Chicago Press : 47-59.

1985 “The Drawings of Onfim”, School Arts 84, March : 6-8.

1990 "The Archeology of Novgorod", Scientific American 262-2 : 84-91.

1997 "Novgorod Birchbark Letters", Anthropology and Archeology of Eurasia 35-4 : 14-41.

Luouet, Georges-Henri

1927 Le Dessin enfantin. Paris, Alcan.
PICARD, Delphine, BrECHET, Claire, et BALDY, René

2007 "Expressive Strategies in Drawing Are Related to Age and Topic", Journal of Nonverbal Behaviour 31-4, Dec. : 243-25?.

Rouzaud, François, MAUduIt, Éric, et CALVET, Jean-Paul

1994 « L'art pariétal médiéval de la grottemine du Calèl demeure menacé. Sorèze, Tarn (France) » (bilingue français-anglais), Lettre internationale d'informations sur l'art rupestre 9, Foix : 14-18.

\section{THOMPson, M. W.}

1967 Novgorod the Great. Excavations at the Medieval City. New York, Frederick A. Praeger ed.

\section{Wickenden of THANET, Paul}

1995 "Medieval Children's Art", Tournaments Illuminated 116, Fall : 19.

2004 "The Art of Onfim: Medieval Novgorod Through the Eyes of a Child". Page web de l'auteur.

\section{ZANGemeister, Carolus}

1875 Corpus Inscriptionum Latinarum, IV, Inscriptiones Parietariae Pompeianae, Herculanenses. Stabianae, Berolini. 